\title{
SOLVENT FREE REGIOSELECTIVE HETEROCYCLIZATION OVER HZSM-5 ZEOLITE UNDER MICROWAVE IRRADIATION SYNTHESIS OF CONDENSED THIAZOLES
}

\author{
Majid M. Heravi ${ }^{a}$, Majid M. Sadeghi ${ }^{b}$, Mohammad Froomand ${ }^{b}$, \\ Shahnaz Khaleghi ${ }^{\mathrm{a}}$ and Mitra Ghassemzadeh ${ }^{c}$ \\ ${ }^{a}$ Department of Chemistry, School of Sciences, Azzahra University, Tehran, Iran \\ bYazd Azad University, Yazd, Iran \\ ${ }^{c}$ Chemistry and Chemical Engineering Research Center of Iran, Tehran, Iran \\ e-mail:mmh1331@yahoo.com
}

\begin{abstract}
Mercapto-1,2,4-triazin-5-one 1 and 3-mercapto-1,2,4-triazoles 2 were condensed with allyl bromide in the presence of base to afford the corresponding 3-allylmercapto compounds 3 and 4 , respectively. These compounds were regioselectively cyclized to give 2,3-dihydro-3,6-dimethylthiazolo[3,2-b][1,2,4]triazine $\mathbf{5}$ and 2,3dihydro-3-methylthiazolo[3,2-b][1,2,4]triazoles 6 respectively.

Keywords: triazole, triazine, thiazole, microwave irradiation, zeolite, solventless system

In our previous papers, we have described the regioselective cyclizations for the synthesis of condensed thiazoles (1). Many efforts have been devoted to the preparation of a variety of heterocyclic system by using Pd (2) or acid (3) catalyzed intramolecular functionalization of olefins as the ring forming step. With a view to extending the synthetic utility of allylic type compounds we have investigated the heterocyclization of 3-allylmercapto-1,2,4-triazine 3 and 3-allylmercapto-1,2,4-triazoles $4 a$ and $b(R=H, M e, P h)$. Compounds 3 (4) and 4 (5) were prepared according to reported procedures. We have recently reported on solid state heterocyclization using zeolite (6), sulfuric acid adsorbed on silica gel (7), and on the catalytic use of natural zeolite in a Biginelli heterocyclization (8).
\end{abstract}

In continuation of our attempts to develop selective and preparatively useful methodology based on the use of solid acids as a promoters (9) and microwave irradiation as a source of energy (10), we have treated compound 3 with HZSM-5 zeolite (11) under microwave irradiation.<smiles></smiles><smiles>C=CCSc1nc(=O)c(N)n[nH]1</smiles><smiles>[R]c1nnc(SCC=C)n1C</smiles>

Scheme-1

The reaction was conducted simply by grinding compound 3 with HZSM-5 zeolite using pestle and mortar and placed the mixture in microwave oven. After work up, a single crystalline compound was obtained. This compound was identified as 2,3-dihydro-3,6-dimethylthiazolo[3,2-b][1,2,4]triazin-7-one 5 by comparison of the physical and spectral data with those of the reported compound (4).

Compound $4 a$ and $4 b(R=H, M e, P h)$ was also treated with HZSM-5 zeolite in the same manner to yield the substituted 2,3-dihydro-3-methyl thiazole[3,2-b][1,2,4]triazoles $\mathbf{6 a}$ or $\mathbf{6 b}$. This compound was identified also by comparison of its physical and spectroscopic data with those of the previously reported compound (5).

In summary we have developed an efficient, simple and fast method for the synthesis of condensed thiazole using inexpensive and available HZSM-5 zeolite.

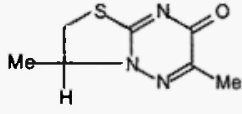

5

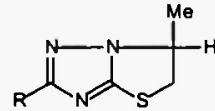

6

Scheme 2

Experimental

Melting points obtained on a Büchi 530 apparatus are uncorrected. 'HNMR spectra were recorded on a Bruker AC 100 spectrometer. IR spectra were recorded on a Perkin-Elmer model 883 using $\mathrm{KBr}$ disc and mass spectra were obtained on a Varian CH-7. 
Synthesis of condensed thiazoles using HZSM-5 zeolite under microwave irradiation in solventless system; General procedure:

Compound 3 or $4(1 \mathrm{mmol})$ was ground with HZSM-5 zeolite thoroughly to make an intimate pair. The mixture was placed in microwave oven for $5 \mathrm{~min}$. The progress of reaction was monitored by TLC. Upon completion of the reaction, the residue was taken into methanol and filtered off. The filtrate was evaporated to dryness to afford the corresponding condensed thiazoles 5 or 6 , respectively.

\section{Selected data for 5}

Yield: $76 \%, \mathrm{mp}: 149-150^{\circ} \mathrm{C}$ (lit. (4): $148-150^{\circ} \mathrm{C}$ ), ${ }^{1} \mathrm{HNMR}, \delta\left(\mathrm{CDCl}_{3}\right) 1.55$ (d, $\left.J=6.0 \mathrm{~Hz}, 3 \mathrm{H}, \mathrm{Me}\right), 2.49(\mathrm{~s}, 3 \mathrm{H}$, Me), 3-3.7 (m, 2H, diastereotopic $\mathrm{CH}_{2}$ ), 4.5-4.8 (m, 1H, CH); IR $\widetilde{v}(\mathrm{KBr}$ disc) 3423, 2929, 1650, 1466, 1373, 1229, $1135,777 \mathrm{~cm}^{-1}, \mathrm{MS}, \mathrm{m} / \mathrm{z}, \mathrm{M}^{+} 183, \mathrm{UV}(\mathrm{EtOH}) \lambda_{\max }: 248 \mathrm{~nm}$.

\section{Selected data for $6(R=H)$}

Yield: $48 \%$, mp: $156-7^{\circ} \mathrm{C}$; ${ }^{1} \mathrm{HNMR}, \delta\left(\mathrm{CDCl}_{3}\right) 1.5(\mathrm{~d}, J=6.0 \mathrm{~Hz}, 3 \mathrm{H}, \mathrm{Me}), 2.2(\mathrm{~s}, 3 \mathrm{H}, \mathrm{Me}), 3.2-3.6(\mathrm{q}, 1 \mathrm{H}$, diastereotopic $\mathrm{CH}$ ), 3.7-4.1 (q, 1H, diastereotopic $\mathrm{CH}$ ), 4.1-4.6 (m, 1H, CH); IR $\widetilde{v}\left(\mathrm{KBr}\right.$ disc) 2980, 1141, $1377 \mathrm{~cm}^{-}$ '; MS, $\mathrm{m} / \mathrm{z}, \mathrm{M}^{+} 155, \mathrm{UV}(\mathrm{MeOH}) \lambda_{\max }: 237 \mathrm{~nm}$.

Selected data for $6\left(\mathrm{R}=\mathrm{CH}_{3}\right)$

Yield: $49 \%, \mathrm{mp}: 158-9^{\circ} \mathrm{C}$; ${ }^{1} \mathrm{HNMR}, \delta\left(\mathrm{CDCl}_{3}\right) 1.5(\mathrm{~d}, J=6.0 \mathrm{~Hz}, 3 \mathrm{H}, \mathrm{Me}), 2.2(\mathrm{~s}, 3 \mathrm{H}, \mathrm{Me}), 3.2-3.6(\mathrm{q}, 1 \mathrm{H}$, diastereotopic $\mathrm{CH}$ ), 3.7-4.1 (q, 1H, diastereotopic $\mathrm{CH}$ ), 4.1-4.6 (m, 1H, CH); IR $\widetilde{v}\left(\mathrm{KBr}\right.$ disc) 2980, $1141,1377 \mathrm{~cm}^{\circ}$ ${ }^{\prime} ; \mathrm{MS}, \mathrm{m} / \mathrm{z}, \mathrm{M}^{+} 155, \mathrm{UV}(\mathrm{MeOH}) \lambda_{\max }: 237 \mathrm{~nm}$.

\section{Selected data for $3(R=P h)$}

Yield: $50 \%$, mp: $79-80^{\circ} \mathrm{C}$; ' $\mathrm{HNMR}, \delta\left(\mathrm{CDCl}_{3}\right) 1.5(\mathrm{~d}, J=6 \mathrm{~Hz}, 3 \mathrm{H}, \mathrm{Me})$, , 3.0-3.5 (q, $1 \mathrm{H}$, diastereotopic $\left.\mathrm{CH}\right), 3.5-$ 4.0 (q, $1 \mathrm{H}$, diastereotopic $\mathrm{CH}), 4.0-4.6(\mathrm{~m}, 1 \mathrm{H}, \mathrm{CH}), 7.5(\mathrm{~s}, 2 \mathrm{H}$, aromatic), $8.0(\mathrm{~s}, 2 \mathrm{H}$, aromatic); IR $\widetilde{v}(\mathrm{KBr}$ disc) $2929,1637,1473,1431 \mathrm{~cm}^{-1} ; \mathrm{MS}, \mathrm{m} / \mathrm{z}, \mathrm{M}^{+} 217, \mathrm{UV}(\mathrm{MeOH}) \lambda_{\max }: 265,238,218 \mathrm{~nm}$.

\section{References}

1. a) M. M. Heravi, K. Aghapoor, and M. A. Nooshabadi, Synth. Commun 28, 233 (1998); b) M. M. Heravi and M. Tajbakhsh, J. Chem. Res. 8, 488 (1998); c) M. M. Heravi, N. Montazeri, M. Bakavoli and M. Ghassemzadeh, J. Chem. Res. 10, 584 (2000); d) M. M. Heravi, A. Keivanloo, M. Rahimizadeh, M. Bakavoli and M. Ghassemzadeh, Tetrahedron Lett. 45, 5747 (2004); e) M. M. Heravi, A. Keivanloo, M. Rahimizadeh, M. Bakavoli, M. Ghassemzadeh and B. Neumüller, Tetrahedron Lett. 46, 1607 (2005).

2. a) M. Mizutani, Y. Sanemitsu, Y. Tamaru and Z. Yoshida, J. Org. Chem. 48, 4585 (1983); b) M. A. Nooshabadi, K. Aghapoor, K. Tabar-Hydar, and M. M. Heravi, Indian J. Heterocyclic Chem. 7, 4 (1998).

3. M. M. Heravi, S. M. Bolourtchian, M. A. Kiakoojori and M. A. Nooshabadi, Indian J. Heterocyclic Chem. 7, 305 (1998).

4. M. M. Heravi, M. A. Nooshabadi and K. Aghapoor, Phosphorus, Sulfur and Silicon 164, 95 (2000).

5. M. M. Heravi, M. Khademolfoghara, M. M. Sadeghi and M. Ghassemzadeh, Phosphorus, Sulfur and Silicon (2005), in press.

6. M. A. Nooshabadi, K. Aghapoor, S. M. Bolourtchian, and M. M. Heravi, J. Chem. Res. 8, 498 (1999).

7. M. M. Heravi, N. Montazeri, M. Rahimzadeh, M. Bakavoli and M. Ghassemzadeh, J. Chem. Res. 10, 584 (2000).

8. M. Tajbakhsh, B. Mohajerani, M. M. Heravi and A. N. Ahmadi, J. Molecular Catalysis A, Chemicals 236, 216 (2005).

9. a) M. M. Heravi, F. Derikvand and F. F. Bamoharram, J. Mol. Catal. A, Chemicals 224, 173(2005); b) M.M.Heravi, Kh. Bakhtiari, Sh. Taheri and H. A. Oskooie, Green Chem. 7, 867 (2005).

10. M. M. Heravi, R. Motamedi, H. A. Oskooie, and M. Ghassemzadeh, Het. Commun. 11, 423 (2005).

11. M. M. Heravi, D. Ajami, K. Aghapoor and M. Ghassemzadeh, Chem.Commun. 833 (1999)

Received on November 30, 2005 